\title{
What stellar populations can tell us about the evolution of the mass-metallicity relation in SDSS galaxies
}

\author{
N. Vale Asari ${ }^{1,2}$, G. Stasińska ${ }^{2}$, R. Cid Fernandes ${ }^{1}$, J. M. Gomes ${ }^{1,3}$, \\ M. Schlickmann ${ }^{1}$, A. Mateus ${ }^{4} \&$ W. Schoenell ${ }^{1}$ \\ ${ }^{1}$ Dpto. de Física - CFM - Universidade Federal de Santa Catarina, Florianópolis, SC, Brazil \\ ${ }^{2}$ LUTH, Observatoire de Paris, CNRS, Université Paris Diderot; Place Jules Janssen 92190 \\ Meudon, France \\ ${ }^{3}$ GEPI, Observatoire de Paris, CNRS, Université Paris Diderot; Place Jules Janssen 92190 \\ Meudon, France \\ ${ }^{4}$ IAG, Universidade de São Paulo, São Paulo, SP, Brazil
}

\begin{abstract}
During the last three decades, many papers have reported the existence of a luminosity metallicity or mass metallicity $(M-Z)$ relation for all kinds of galaxies: The more massive galaxies are also the ones with more metal-rich interstellar medium. We have obtained the massmetallicity relation at different lookback times for the same set of galaxies from the Sloan Digital Sky Survey (SDSS), using the stellar metallicities estimated with our spectral synthesis code STARLIGHT. Using stellar metallicities has several advantages: We are free of the biases that affect the calibration of nebular metallicities; we can include in our study objects for which the nebular metallicity cannot be measured, such as AGN hosts and passive galaxies; we can probe metallicities at different epochs of a galaxy evolution.

We have found that the $M-Z$ relation steepens and spans a wider range in both mass and metallicity at higher redshifts for SDSS galaxies. We also have modeled the time evolution of stellar metallicity with a closed-box chemical evolution model, for galaxies of different types and masses. Our results suggest that the $M-Z$ relation for galaxies with present-day stellar masses down to $10^{10} M_{\odot}$ is mainly driven by the star formation history and not by inflows or outflows.
\end{abstract}

Keywords. galaxies: evolution, galaxies: statistics, galaxies: stellar content.

A full version of this study is presented in Vale Asari et al. (2009).

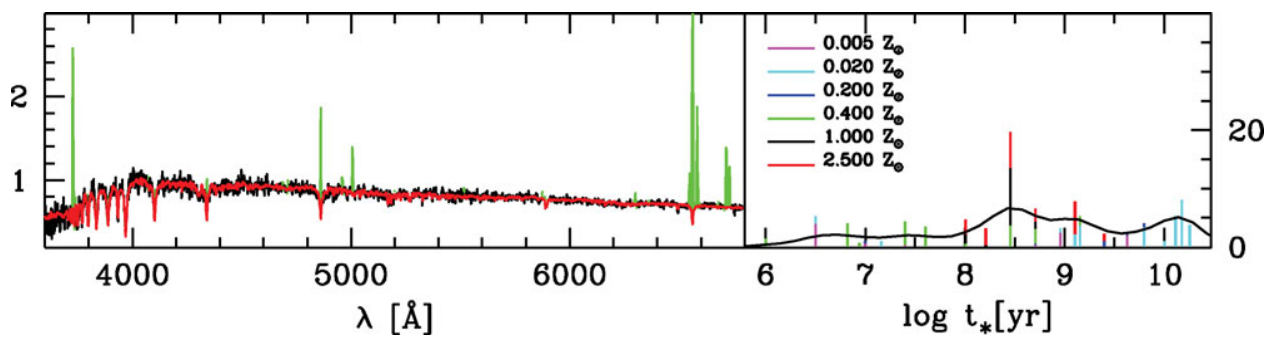

Figure 1. We have processed 573141 SDSS galaxies with our pixel-by-pixel STARLIGHT algorithm, which fits a galaxy spectrum with a sum of simple stellar populations (SSPs) of different ages and metallicities; Left panel shows an example of observed (black+green) and model (red) spectra. Emission lines (green) are masked out. Right panel shows the light fraction (in percents) associated with the SSPs. See Cid Fernandes et al. (2005) for more details about the method. 

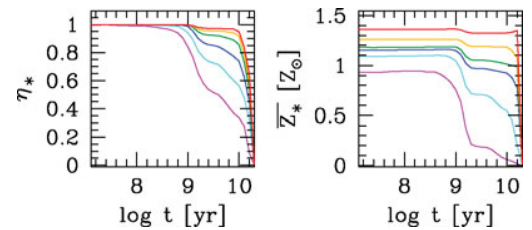

Figure 2. From the ages and metallicities of the SSPs in a galaxy we recover histories of stellar mass and stellar metallicity. Our sample has been divided into six present-day stellar mass bins centered in $\log M_{\star} / M_{\odot}=10.0$ (A-magenta), 10.3 (B-cyan), 10.6 (C-blue), 10.9 (D-green), 11.2 (E-yellow) and 11.5 (F-red), each one 0.30 dex wide. Panels show the cumulative stellar mass history $\left(\eta_{\star}(t) \equiv M_{\star}(t) / M_{\star}(t=0)\right.$, left $)$ and the mean stellar metallicity $\left(\overline{Z_{\star}}(t)\right.$, right $)$ as a function of lookback time $t$ for bins $\mathrm{A}-\mathrm{F}$. We find that the more massive a galaxy is today, the faster it has formed stars and produced metals.

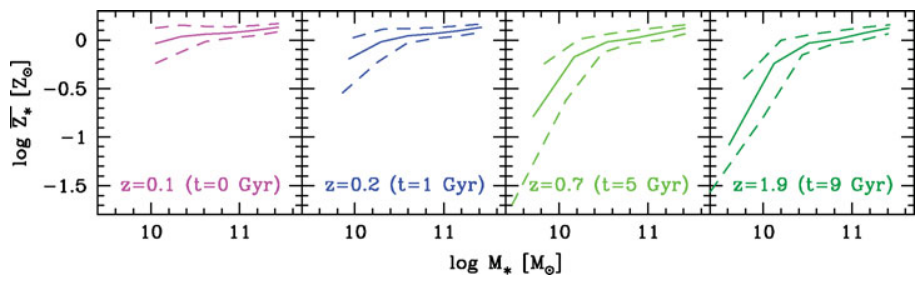

Figure 3. Given $\eta_{\star}(t)$ and $\overline{Z_{\star}}(t)$ for a set of galaxies, another way to study their evolution is to look at a snapshot of the $M_{\star}-Z_{\star}$ relation for a given lookback time $t$. Solid lines mark the median and dashed lines the quartiles of the distribution. As lookback time increases, the $M_{\star}-Z_{\star}$ relation steepens and covers a larger range of values. The novelty is that we are looking at the same set of galaxies in each lookback time. That was only possible because we have derived stellar instead of nebular metallicities.

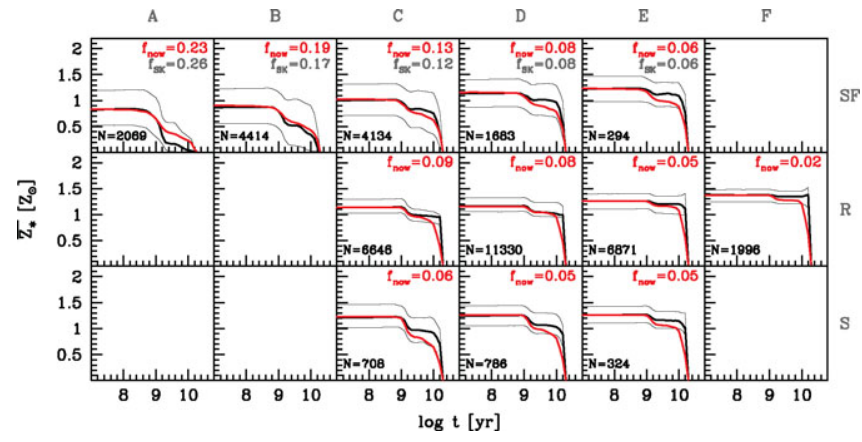

Figure 4. We show a simple closed-box chemical evolution model for mass-bins A-F and by galaxy type: star-forming (SF), retired/passive (R) and Seyfert (S). Each panel shows the median and quartiles of the evolution of $Z_{\star}$ as found by STARLIGHT (black lines) and as obtained with the simple closed-box model (red lines). The value of the present-day gas fraction $\left(f_{\text {now }}\right)$ needed to reproduce the median present-day $Z_{\star}$ is indicated at the top right of each panel. For the SF sample, we also indicate the median of $f_{\text {now }}$ as measured by the Schmidt-Kennicutt law $\left(f_{\mathrm{SK}}\right)$. This suggests that the $M_{\star}-Z_{\star}$ relation for galaxies with present-day stellar masses down to $10^{10} M_{\odot}$ is mainly driven by the star formation history.

\section{References}

Cid Fernandes, R., Mateus, A., Sodré, L., Stasińska, G., \& Gomes, J. M. 2005, MNRAS, 358, 363

Vale Asari, N., Stasińska, G., Cid Fernandes, R., Gomes, J. M., Schlickmann, M., Mateus, A., \& Schoenell, W. 2009, MNRAS, 396, L71 